\title{
Current perspectives in stem cell therapy for spinal cord repair in humans: a review of work from the past 10 years
}

\author{
Atuais perspectivas da terapia com células tronco para regeneração medular em \\ humanos: um revisão de trabalhos dos últimos 10 anos \\ Eric Domingos Mariano 1,3, Chary Marquez Batista,3, Breno José Alencar Pires Barbosa1, Suely Kazue \\ Nagahashi Marie ${ }^{1,3}$, Manoel Jacobsen Teixeira ${ }^{1}$, Matthias Morgalla², Marcos Tatagiba², Jun Li², \\ Guilherme Lepski i,2,3
}

\begin{abstract}
Spinal cord injury (SCI) and amyotrophic laterals sclerosis (ALS) are devastating neurological conditions that affect individuals worldwide, significantly reducing quality of life, both for patients and their relatives. Objective: The present review aims to summarize the multiple restorative approaches being developed for spinal cord repair, the use of different stem cell types and the current knowledge regarding stem cell therapy. Method: Review of the literature from the past 10 years of human studies using stem cell transplantation as the main therapy, with or without adjuvant therapies. Conclusion: The current review offers an overview of the state of the art regarding spinal cord restoration, and serves as a starting point for future studies.
\end{abstract}

Keywords: spinal cord injury, amyotrophic lateral sclerosis, stem cells, stem cell therapy.

\section{RESUMO}

Lesão medular (LM) e esclerose lateral amiotrófica (ELA) são condições devastadoras que acometem pessoas em todo o mundo, reduzindo a qualidade de vida tanto de pacientes como de entes queridos. Objetivo: A atual revisão tem como alvo as múltiplas abordagens restauradoras para a regeneração medular, o uso de diferentes tipos celulares e o atual conhecimento a cerca da terapia com células tronco. Método: Revisão de literatura dos últimos 10 anos usando transplantes de células tronco como estratégia principal, com ou sem terapia adjuvante, em humanos. Conclusão: A presente revisão oferece uma visão geral acerca da restauração medular e serve de ponto de partida para estudos futuros.

Palavras-chave: lesão medular, esclerose lateral amiotrófica, células tronco, terapia com células tronco.

For a long time, the spinal cord was seen as a tube that merely connected the brain to different organs and structures in the human body. This simplified view changed thanks to the brilliant contribution of Sir Charles S. Sherrington, whose famous monograph "The Integrative Action of the Nervous System", explained how the (CNS) is organized. With this monograph, Sherrington settled the long-standing debate between "Reticular Theory" (which argued that neurons are physically contiguous) and the "Neuron Doctrine" (which suggested neurons communicate with each other via synapses) ${ }^{1}$. Another strong belief at the time was that the CNS was incapable of regenerating. This view was challenged when Santiago Ramón y Cajal showed that the transected spinal cords of animals were indeed able to regenerate; however, this spontaneous regeneration lasted only for about 10 to 14 days $^{2}$.

In this paper, we review the ways in which stem cell therapy can promote spinal cord regeneration in human subjects. We have chosen as our focus two different pathologies: Spinal Cord Injury (SCI) and Amyotrophic Lateral Sclerosis (ALS).

Spinal Cord Injury (SCI) is a devastating disease with an incidence of 12,000 new cases per year in the United States alone. According to the National Spinal Cord Injury Statistical

${ }^{1}$ Departamento de Neurologia, Faculdade de Medicina, Universidade de São Paulo, Sao Paulo SP, Brazil;

${ }^{2}$ Department of Neurosurgery, Eberhard-Karls University, Tuebingen, Germany;

${ }^{3}$ Laboratório de Biologia Molecular e Celular, Universidade de São Paulo, Sao Paulo SP, Brazil.

Correspondence: Guilherme A. Lepski; Av. Dr. Arnaldo, 455 Cerqueira Cesar, LIM 15 - $4^{\circ}$ andar; 01246-903 São Paulo SP, Brasil; E-mail: lepski@usp.br

Conflict of interest: There is no conflict of interest to declare.

Received 16 December 2013; Received in final form 26 March 2014; Accepted 16 April 2014. 
Center $^{3}$, the major causes of SCI are automobile accidents (41.7\%), falls (27.3\%), violence (15\%) and sports $(7.8 \%)$.

An SCI lesion is essentially acute ischemia that causes injury to vertebral bones, ligaments and discs. Usually, it is caused by a contusion or compression. The concentration of toxic variables and the ischemia itself contribute to cell death and necrosis, which may cause secondary lesions that occur at the cellular level and are often more complex. By blocking action potentials, deregulating the ion balance, causing lipid peroxidation and glutamatergic excitotoxicity, these secondary lesions can cause functional disturbances such as central sensitization, cell death, necrosis, axonal damage and swelling. These noxious events may in turn lead to other pathologies, such as chronic neuropathic pain ${ }^{4}$.

Immediately following injury, astrocytes play an important role in glial scar formation ${ }^{4}$. Glial scarring stimulates axonal regeneration; following a CNS injury, it isolates the nerve tissue from inflammatory cells, thus supporting physical and chemical integrity. On the other hand, glial scarring is also responsible for preventing axonal invasion, thus hindering neuroregeneration. Although glial scarring is an important contributor to regeneration, few axons can cross the glial scar as early as four weeks after an $\mathrm{SCI}^{4}$.

Amyotrophic lateral sclerosis (ALS); (also known as Charcot disease, motor neuron disease and Lou Gherig's disease) is a neurodegenerative disease in which a progressive paralysis leads to death, frequently by respiratory failure, within 3-5 years of the first symptoms. Only 5\% of patients with ALS have a family history, but an extensive family history usually increases the chances of developing ALS ${ }^{5}$. ALS begins focally and spreads contiguously. It may first appear as paralysis in one hand or leg, or as dysarthria or dyspnea, and then evolves progressively to other body regions, causing classic lower motor neuron symptoms. As neurons of the anterior horn die, motor axons are lost, causing a reduction in the amplitude of muscle action potentials. Electromyography (EMG) is thus a useful tool to diagnose ALS and exclude other pathologies, such as other motor neuron diseases, peripheral neuropathies and neuromuscular disorders ${ }^{6}$.

ALS occurs in approximately 2 out of every 100,000 people per year. The most disturbing feature of this condition is that when the first symptoms appear, the pathology is already well underway ${ }^{6}$. It is difficult to determine which factors contribute to degeneration and which factors are just consequences of the cellular adaptation caused by the condition itself. However, it is well-established that excessive glutamate, astrocyte dysfunction, mitochondrial deficits, and nuisance in protein degradation, are some key features of this condition ${ }^{5}$.

It is reasonable to assume that any injury to the spinal cord will result in serious consequences, whether it is caused by trauma or by a genetic predisposition. One promising method of regenerating the CNS is stem cell transplantation. Much of the current research in this field of study is dedicated to identifying the type of cell that can promote regeneration in the safest and most effective way. Other than cell type, features that are important to the functional and structural restoration of impaired neurons are (1) electrophysiological properties, (2) synaptogenesis and (3) interaction with the host tissue ${ }^{7}$. The main objective of cell transplantation is to promote neural regeneration and functional neural recovery by enhancing the local regenerative capacity with neurotrophic factors. This promotes axonal regeneration and replaces lost neurons ${ }^{8}$.

\section{SCHWANN CELLS}

Schwann cells (SC) are responsible for producing the myelin sheath and guidance bands used in remyelination and axonal regeneration after injury. When transplanted, Schwann cells produce several neurotrophic factors (such as NGF, BDNF, and CNTF) that contribute to neuronal survival and to the generation of cell adhesion molecules and extracellular matrix proteins that support axonal growth. Glial scars, a potent natural barrier, represent an obstacle for SC migration, as do chondroitin sulfate proteoglycan (CSPG), aggrecan and ephrins ${ }^{8,9}$.

In a comparative study with olfactory ensheathing cells (OECs), it was observed that SCs interact with astrocytes, causing them to proliferate and grow. Furthermore, the myelinated axons were separated into groups. There was a significantly enhanced expression of glial fibrillary acidic protein (GFAP) - about 5.95\% more than was seen for OECs 3 weeks post injection and about $3.71 \%$ more than was seen for OECs 6 weeks after the injection. SC migration was more concentrated at the site of injury, but the OECs "walked" a greater distance, and even penetrated the normal tissue. Although both cell types were able to effect functional recovery, OECs were more successful at it ${ }^{10}$.

In a longitudinal study conducted with human participants in China, investigators tested the transplantation of SCs in six patients and observed the results for over 5 years. At the end of the study, they reported autonomic, sensory, and motor improvement in all patients. MRI scans revealed that the myelomalacia and cystic degeneration had also been reduced. Although this study's results were promising, it had some limitations: patients were not matched for variables such as age, time since SCI, and ASIA scale ${ }^{11}$. In two other longitudinal studies, Saberi et al. aimed to evaluate the safety and feasibility of SC transplantation. In both studies, there were no significant changes in patients' MRI scans, and they reported neither neurological worsening nor improvement. They only reported autonomic functional recovery and improved quality of life $\mathrm{e}^{12,13}$. 


\section{EMBRYONIC STEM CELLS}

Embryonic stem cells (ESCs) are a type of pluripotent cell found in the blastocyst, which is capable of differentiating in the 3 primary gem layers and generating all cell types, making these cells the perfect candidates for cell therapy. Neural differentiated ESCs may develop into oligodendrocytes, astrocytes or neurons ${ }^{14}$. There are ethical considerations surrounding the use of human ESCs, as obtaining them requires the destruction of several human embryos or fertilized oocytes.

A major concern about the use of ESCs is the formation of tumors, such as teratomas. In their study, Matsuda et al. reported tumor formation 21 days after transplantation when all behavioral improvements had ceased. They were then able to suppress tumorigenesis in a co-culture with bone marrow stromal cells (BMSC) ${ }^{15}$.

Despite the fear of tumor formation, scientists at the Geron Corporation initiated clinical trials using ESC-derived OPCs (GRNOPC1) administered within 14 days of injury. No adverse events were reported at the time of the longterm follow-up ${ }^{8}$.

\section{MESENCHYMAL STEM CELLS / BONE MARROW STEM CELLS}

The bone marrow may be the only organ where two types of stem cells can be found coexisting and cooperating. The BMSCs (or hematopoietic stem cells (HSCs)), produce blood cells, while mesenchymal stem cells/mesenchymal stromal cells (MSCs or BMSCs), can be found in the stromal layer adjacent to hematopoietic cells. The stroma supports hematopoietic cells, regulating the micro-environment and facilitating the maturation of blood cells. MSCs can also be isolated from other regions such as adipose tissue, neonatal tissue (such as umbilical cord and placenta), and fetal tissues like lungs, liver and blood ${ }^{16}$.

In 2006, Moviglia et al. reported a combined treatment with MSCs and autoimmune T (AT) cells with 2 spinal cord injury patients. The main goal behind this approach is to control inflammatory activity in order to create the ideal microenvironment for cell transplantation, as it has been shown that AT cells are essential for tissue repair. Both patients reported motor improvement and no adverse effects ${ }^{17}$.

In a clinical trial, Saito et al. described a single patient who had a fracture-dislocation at C5 and reported loss of sensation and movement below the lesion site. The investigators transplanted autologous BMSCs by lumbar puncture 13 days after injury and evaluated the patient for 6 months. They were able to evidence some motor and sensory improvement (relative to pre-transplantation scores) after
1-3 months; after 6 months, they observed a slight motor improvement with no further sensory improvement. They continued to accompany the patient after the 6-month period and reported that he was able to sit in a wheelchair and even drive it slowly ${ }^{18}$. Then, the same group conducted another study with the patient studied previously and 4 new ones who had severe spinal cord lesions and were still in the acute phase (up to 72 hours after injury). Following the same 6 -month observation period, 4 of the 5 patients experienced motor improvement. The fifth patient did not show motor improvement, yet his condition stayed stable during the entire trial period. Importantly, 3 of the patients showed good results as early as 6 months after transplantation $^{19}$.

In a study by Pal et al. 30 patients were divided into two groups: 20 patients who had been injured for less than 6 months and 10 who had suffered injury for more than 6 months. Patients ranged in age between 18-53 years, and their injuries were between C4-T10. The authors transplanted autologous MSCs via lumbar puncture. No adverse effects were reported. There were no significant differences in the MRI scans taken at baseline and at the 1-year follow-up. Similarly, somatosensory evoked potentials (SSEPs), motor evoked potentials (MEPs) and nerve conduction velocity (NCV) measurements revealed no significant changes. Despite this negative electrophysiological finding, patients did indeed report significant recovery: initially in bladder sensation, followed by bladder regulation and bowel function, then improvement in the sensory tract, and finally in motor function ${ }^{20}$.

In a study by Ra et al., 8 male patients who had suffered from spinal cord injury for more than 12 months agreed to be submitted to an autologous transplantation of MSCs derived from adipose tissue (via the cephalic vein). As a group, these patients reported several adverse effects following transplantation: chest pain and tightness, mild fever, furuncle on the upper thigh, musculoskeletal pain, painful neck and shoulder, increased sputum, upper respiratory infection, urinary incontinence, urinary tract infection, aggravation of spasticity, neuropathic pain, pain exacerbation, headache, low thyroid stimulating hormone and somnolence. On the other hand, their electrophysiological (SSEP and MEP) recordings were not significantly different than before transplantation. However, following treatment, one patient who previously had problems feeding and dressing himself was able to hold a cup and dress with partial assistance ${ }^{21}$. In another study comparing MSC transplantation to conventional treatment, confirmed the safety and feasibility of using this technique via lumbar puncture; however, even though some recovery was reported, it was not found to be statistically significant ${ }^{22}$.

Vercelli et al. reported that human mesenchymal stem cells (MSCs) could survive and migrate in the lumbar spinal 
cord of SOD1 mice (ALS animal model), prevent astrogliosis and microglial activation, and delay the reduction in the number of motoneurons ${ }^{23}$. A more recent study comparing $\mathrm{BMC}$ to bone marrow-derived MSCs reported that BMCs were more effective at restoring motor function and also had a higher survival rate ${ }^{24}$.

Mazzini et al. followed 19 patients (11 males and 8 females ages 20-75) from November 2001 to September 2003. The number of months from the time of diagnosis to recruitment ranged from 3-81. The goal of the study was to evaluate the safety and feasibility of MSC transplantation. All patients were monitored for serious adverse effects (e.g., tumors, aberrant connections from MSC transplantation, and death). Patients were evaluated with MRI and tractography, which revealed no new masses at the site of injection or in the neuraxis, no syringomyelia or pseudomeningocoele, and no alterations in the corticospinal tracts. When asked about quality of life, their responses did not differ significantly from those given at the beginning of the study ${ }^{25}$. In another study by Karussis et al., 34 patients (15 with multiple sclerosis (MS) and 19 with ALS) received MSC via lumbar puncture, and 14 also received intravenous MSC injections. Fifteen patients in this group experienced headaches associated with the lumbar puncture that lasted for up to 7 days. Other than that, none of them reported any acute or chronic adverse effects. These results provide supporting evidence for the immunomodulating effects of MSC transplantation ${ }^{26}$.

In another study with 13 patients, Deda et al. performed a laminectomy at level C1-C2, and injected BMSCs into the anterior part of patients' spinal cords. The EMG analysis revealed signs of re-innervation, pre- and postoperatively, in 9 patients. At the 1-year follow-up, these patients showed a slight neurological regression, although they were still better relative to before the study ${ }^{27}$. Finally, in another study that took place over one year, Blanquer et al. monitored the neuroprotective effects of BMSC nest transplants. Results showed that the number of motoneurons increased and were surrounded by CD90+ cells, with no presence of degenerative ubiquitin deposits in the treated segments ${ }^{28}$.

\section{OLFACTORY ENSHEATHING CELLS}

Neurogenesis in the olfactory system continues to take place throughout a person's life. Stem cells proliferate to generate new sensory neurons in the basal layer of the olfactory epithelium. Within the central nervous system, stem cells proliferate in the subventricular zone of the forebrain, generating neural progenitors that migrate to the olfactory bulb to create new interneurons. In case of injury, these neurons are immediately replaced through a surge in neurogenesis. Their axons grow out through the basal layer of the epithelium, penetrate the basement membrane and enter the laminae propria, forming axon bundles that are ensheathed by OECs. These bundles penetrate the skull and reach the olfactory bulb, where they form synapses with mitral cells and interneurons. OECs surround the axons of the sensory neurons in the olfactory epithelium and form synapses in the specialized glomeruli of the olfactory bulb in the brain. Due to their ability to guide the connections between the peripheral nervous system (PNS) and the CNS, and to their ability to differentiate into non-olfactory cell types, these multipotent cells are excellent candidates for cell transplantation ${ }^{29}$.

Huang et al. selected 35 patients and divided them into 2 groups: a control group $(\mathrm{n}=20)$ and an experimental group $(n=15)$ that received OECs derived from fetal olfactory bulb. The experimental group did not report any adverse effects, suggesting that OEC transplantation may slow disease progression $^{30}$. In another study with 42 patients, 35 received OEC transplants 2 times, 5 received transplants 3 times, 1 received transplants 4 times, and another received them 5 times. The authors reported that all patients recovered neurological function, and none experienced side effects such as tumors, hemorrhages, edema, cyst formations, infections, or disruption of neural structures. Also, 35 patients showed improvements in EMG, and 1 patient showed pulmonary function improvement (but only after the fourth transplant, and remaining stable even after the fifth transplant) ${ }^{31}$.

\section{NEURAL STEM CELLS}

First described by Altman in 1960, these multipotent cells have the potential to become any cell type in the CNS. Neural stem cells are remnants from the neuroectoderm of early embryos and are present in embryonic, fetal and adult nervous systems. During development, these cells divide and differentiate to form the main components of the CNS, i.e., the brain and the spinal cord. During adulthood, stem cells decrease in number and become confined to specific regions, such as the spinal cord, and to a greater extent, the subventricular zone (SVZ) and subgranular zone of the hippocampal dentate gyrus (SGZ) ${ }^{32}$.

Between 2010 and 2011, Glass et al. recruited 12 patients to test the safety of NSC transplantation. They divided patients into 4 groups: A1 and A2 (nonambulatory patients) and $\mathrm{B}$ and $\mathrm{C}$ (ambulatory patients). Groups $\mathrm{Al}$ and $\mathrm{B}$ received 5 unilateral injections of NSCs, while groups A2 and $\mathrm{C}$ received 5 bilateral injections (10 injections total). The investigators performed a laminectomy at T11-T12 and injected the cells. Only adverse effects related directly to the injection procedure were reported. No positive or negative results were obtained, but the authors deemed the trial successful, as it demonstrated the safety of the procedure ${ }^{33,34}$. 
Moviglia et al. described a different strategy in which they used a T-cell vaccine with a technique described as BEN (bone marrow mesenchymal stroma cells, effector $\mathrm{T}$ cells, and neuroblasts). This combined therapy aims to reconstruct the immunological conditions that were present prior to repair (i.e., during the acute phase), to ensure the proper implantation of neuroblasts. None of the 7 patients treated reported side effects, and 1 patient showed some improvement in vital functions. There were no significant signs of motor recovery. The authors concluded that the technique is feasible and safe ${ }^{35}$.

\section{FINAL REMARKS}

The field of stem cell therapy and neural repair has seen important progress in recent years. Abundant pre-clinical data support the notion that cell transplantation promotes repair in the SCI animal model. However, more work must be done to determine (a) the cell type that is most suitable for transplantation, (b) the cell type that has the highest neurogenic potential and, most importantly, (c) the cell type or transplantation technique capable of overcoming the hostile microenvironment and facilitating the restoration of damaged neural tissue.

Naturally, biological safety must be ensured before including stem cell-based treatments in the clinical armamentarium. For instance, one must consider a given stem cell's tendency to generate tumors. Any form of genetic manipulation in which cells are converted to more primitive states triggers higher proliferation rates and therefore carries a higher potential for malignancy. Lentiviral transfections and nuclear transfer assays performed with mature somatic cells solve the problem of stem cell source; however, they do not solve the problem of tumor formation. Therefore, although promising, these techniques are not yet ready for widespread use.

Another promising approach that is currently under development is the recruitment of autologous stem cells, which can be driven to differentiate into mature neurons in order to repopulate damaged areas. With this technique, the cells themselves contain the knowledge about the factors and cell signaling cascades that promote neuronal differentiation and eventually, restoration.

Finally, the reorganization of local neural circuits, aided by the repopulation of the damaged tissue with newly generated neurons (either implanted or recruited locally) is crucial for restoring neural function. The mechanisms beyond this process, and ways to interfere with it, remain largely unknown and require further clarification.

Perhaps the next step in this line of research should be to use combinations of different stem cell transplants, since it has been shown that each cell type is able to recover only a part of the lost tissue. In other words, no single cell type alone can recover an entire region composed of different cells with different functions. Using a combination of different cells would allow the individual action of each cell type to potentiate the activity of the other cells.

In conclusion, the scientific progress of the last several years has contributed enormously to our understanding of neural repair and its potential applications. We have seen promising results in work with animals and in initial clinical trials with human participants. Nevertheless, there is still much to understand about stem cell biology and the pathophysiology of neurodegenerative diseases. We can see the light at the end of the tunnel, and based on the rapid development in recent years, this light is unlikely to fade.

\section{References}

1. Burke RE. Sir Charles Sherrington's the integrative action of the nervous system: a centenary appreciation. Brain 2007;130:887-894.

2. Puchala E, Windle WF. The possibility of structural and functional restitution after spinal cord injury. A review. Exp Neurol 1977;55:1-42.

3. NSCISC, The 2010 Annual Statistical Report for the Spinal Cord Injury Model Systems. 2010: Birmingham, Alabama.

4. Leal-Filho MB. Spinal cord injury: from inflammation to glial scar. Surg Neurol Int 2011;2:112.

5. Al-Chalabi A, Jones A, Troakes C, et al. The genetics and neuropathology of amyotrophic lateral sclerosis. Acta Neuropathol 2012;124:339-352.

6. Sorenson EJ. The electrophysiology of the motor neuron diseases. Neurol Clin 2012;30:605-620.

7. Liebau S, Vaida B, Storch A, Boeckers TM. Maturation of synaptic contacts in differentiating neural stem cells. Stem Cells 2007;25:1720-1729.

8. Li J, Lepski G. Cell transplantation for spinal cord injury: a systematic review. Biomed Res Int 2013;2013:786475.
9

Afshari FT, Kwok JC, Fawcett JW. Astrocyte-produced ephrins inhibit schwann cell migration via VAV2 signaling. J Neurosci 2010;30:4246-4255.

10. Li BC, Xu C, Zhang JY, Li Y, Duan, ZX. Differing schwann cells and olfactory ensheathing cells behaviors, from interacting with astrocyte, produce similar improvements in contused rat spinal cord's motor function. J Mol Neurosci 2012;48:35-44.

11. Zhou XH, Ning GZ, Feng SQ, et al. Transplantation of autologous activated schwann cells in the treatment of spinal cord injury: six cases, more than five years of follow-up. Cell Transplant 2012;21 (Suppl1):S39-47.

12. Saberi H, Firouzi M, Habibi Z, et al. Safety of intramedullary schwann cell transplantation for postrehabilitation spinal cord injuries: 2-year follow-up of 33 cases. J Neurosurg Spine 2011;15:515-525.

13. Saberi H, Moshayedi P, Aghayan HR, et al. Treatment of chronic thoracic spinal cord injury patients with autologous schwann cell transplantation: an interim report on safety considerations and possible outcomes. Neurosci Lett 2008;443:46-50. 
14. McDonald JW, Liu XZ, Qu Y, et al. Transplanted embryonic stem cells survive, differentiate and promote recovery in injured rat spinal cord. Nat Med 1999;5:1410-1412.

15. Matsuda R, Yoshikawa M, Kimura $H$, et al. Cotransplantation of mouse embryonic stem cells and bone marrow stromal cells following spinal cord injury suppresses tumor development. Cell Transplant 2009;18:39-54.

16. Wislet-Gendebien S, Laudet E, Neirinckx V, Rogister B. Adult bone marrow: which stem cells for cellular therapy protocols in neurodegenerative disorders? J Biomed Biotechnol 2012;2012:601560.

17. Moviglia GA, Fernandez Vina R, Brizuela JA, et al. Combined protocol of cell therapy for chronic spinal cord injury. Report on the electrical and functional recovery of two patients. Cytotherapy 2006;8:202-209.

18. Saito F, Nakatani T, Iwase M, et al. Spinal cord injury treatment with intrathecal autologous bone marrow stromal cell transplantation: the first clinical trial case report. J Trauma 2008;64:53-59.

19. Saito F, Nakatani T, Iwase M, et al. Administration of cultured autologous bone marrow stromal cells into cerebrospinal fluid in spinal injury patients: a pilot study. Restor Neurol Neurosci 2012;30:127-136.

20. Pal R, Venkataramana NK, Bansal A, et al. Ex vivo-expanded autologous bone marrow-derived mesenchymal stromal cells in human spinal cord injury/paraplegia: a pilot clinical study. Cytotherapy 2009;11:897-911.

21. Ra JC, Shin IS, Kim SH, et al. Safety of intravenous infusion of human adipose tissue-derived mesenchymal stem cells in animals and humans. Stem Cells Dev 2011;20:1297-1308.

22. Karamouzian S, Nematollahi-Mahani SN, Nakhaee N, Eskandary H. Clinical safety and primary efficacy of bone marrow mesenchymal cell transplantation in subacute spinal cord injured patients. Clin Neurol Neurosurg 2012;114:935-939.

23. Vercelli A, Mereuta OM, Garbossa D, et al. Human mesenchymal stem cell transplantation extends survival, improves motor performance and decreases neuroinflammation in mouse model of amyotrophic lateral sclerosis. Neurobiol Dis 2008;31:395-405.

24. Pastor D, Viso-Leon MC, Jones J, et al. Comparative effects between bone marrow and mesenchymal stem cell transplantation in GDNF expression and motor function recovery in a motorneuron degenerative mouse model. Stem Cell Rev 2012;8:445-458.
25. Mazzini L, Mareschi K, Ferrero I, et al. Mesenchymal stromal cell transplantation in amyotrophic lateral sclerosis: a long-term safety study. Cytotherapy 2012;14:56-60.

26. Karussis D, Karageorgiou C, Vaknin-Dembinsky A, et al. Safety and immunological effects of mesenchymal stem cell transplantation in patients with multiple sclerosis and amyotrophic lateral sclerosis. Arch Neurol 2010;67:1187-1194.

27. Deda H, Inci MC., Kurekci AE, et al. Treatment of amyotrophic lateral sclerosis patients by autologous bone marrow-derived hematopoietic stem cell transplantation: a 1-year follow-up. Cytotherapy 2009;11:18-25.

28. Blanquer M, Moraleda JM, Iniesta F, et al. Neurotrophic bone marrow cellular nests prevent spinal motoneuron degeneration in amyotrophic lateral sclerosis patients: a pilot safety study. Stem Cells 2012;30:1277-1285.

29. Mackay-Sim A, St John JA. Olfactory ensheathing cells from the nose: clinical application in human spinal cord injuries. Exp Neurol 2011;229:174-180.

30. Huang $H$, Chen $L, X i H$, et al. Fetal olfactory ensheathing cells transplantation in amyotrophic lateral sclerosis patients: a controlled pilot study. Clin Transplant 2008;22:710-718.

31. Chen L, Chen D, Xi H, et al. Olfactory ensheathing cell neurorestorotherapy for amyotrophic lateral sclerosis patients: benefits from multiple transplantations. Cell Transplant 2012;21(Suppl1):S65-S77.

32. Andressen C. Neural stem cells: from neurobiology to clinical applications. Curr Pharm Biotechnol 2013;14:20-28.

33. Glass JD, Boulis NM, Johe K, et al. Lumbar intraspinal injection of neural stem cells in patients with amyotrophic lateral sclerosis: results of a phase I trial in 12 patients. Stem Cells 2012;30:1144-1151.

34. Riley J, Federici T, Polak M, et al. Intraspinal stem cell transplantation in amyotrophic lateral sclerosis: a phase I safety trial, technical note, and lumbar safety outcomes. Neurosurgery 2012;71:405-416.

35. Moviglia GA, Moviglia-Brandolino MT, Varela GS, et al. Feasibility, safety, and preliminary proof of principles of autologous neural stem cell treatment combined with T-cell vaccination for ALS patients. Cell Transplant 2012;21(Suppl1):S57-S63. 